\title{
Concepciones sobre enseñanza, aprendizaje, teatro y la práctica en docentes universitarios de la Licenciatura en Artes Escénicas de la Universidad Antonio Nariño
}

\author{
Conceptions about teaching, learning, theater and \\ practice in university teachers of the Bachelor of \\ Performing Arts at the Antonio Nariño University
}

Gabriel Armando Jaramillo Moreno* y John Jairo Briceño Martínez ${ }^{* * *}$

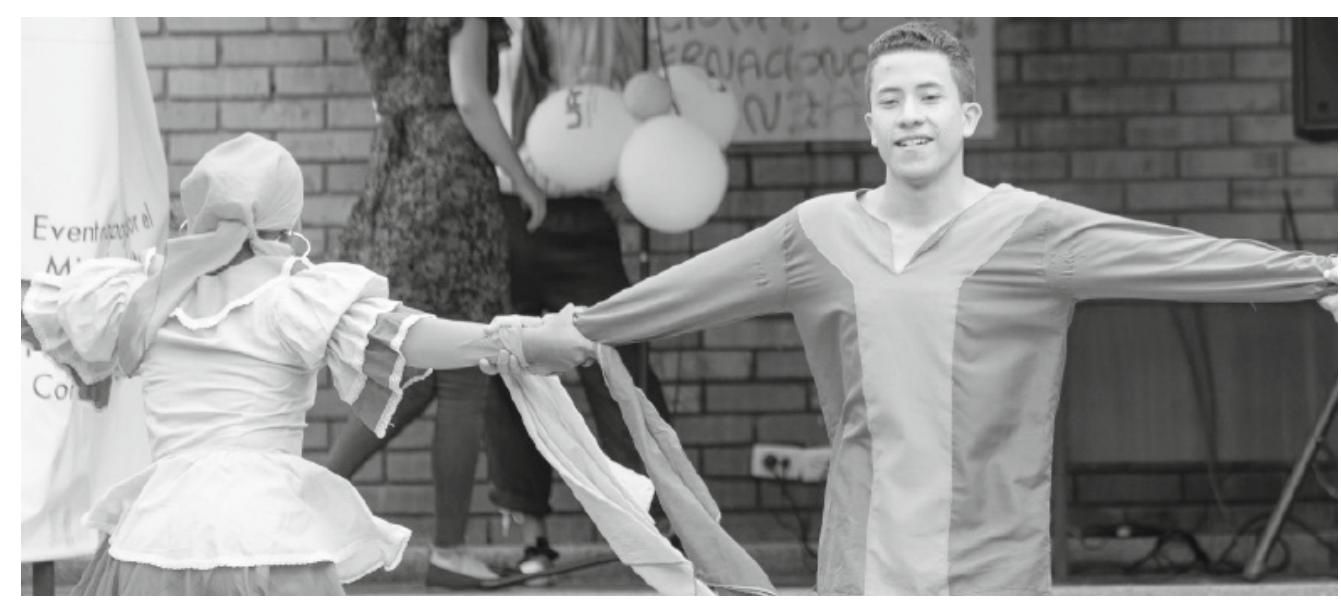

\section{Resumen}

Esta investigación gira en torno a las concepciones de enseñanza, aprendizaje y teatro en tres docentes de la Licenciatura en Artes Escénicas de la Universidad Antonio Nariño, ubicada en Bogotá, Colombia. En vista de que se encuentran pocas investigaciones frente al tema con profesores de esta disciplina, surge la necesidad de profundizar y construir nuevos referentes que aporten a este campo de estudio. En ese sentido, se realiza un análisis de las concepciones y prácticas de los docentes implicados. La investigación es cualitativa y el tratamiento de los datos se realiza mediante el análisis

Citar este artículo como: Jaramillo Moreno, G. A. y Briceño Martínez, J. J. (2020). Concepciones sobre enseñanza, aprendizaje, teatro y la práctica en docentes universitarios de la Licenciatura en Artes Escénicas de la Universidad Antonio Nariño. Revista Papeles, 12(23), 70-85.

Fecha de recibido: 5 de marzo del 2020 Fecha de aprobado: mayo 15 de 2020

* Docente de Bienestar Universitario de la Universidad Antonio Nariño. Correo electrónico: gajaramillo@uan. edu.co

*: Decano de la Facultad de Educación de la Universidad Antonio Nariño. Miembro del Grupo de Investigación Culturas Universitarias. Doctor en Tendencias y Aplicaciones de la Investigación Educativa por la Universidad de Granada (España). Correo electrónico: decano.educacion@uan.edu.co 
de frecuencias que son producto de la codificación realizada. Se concluye que se evidencia una riqueza teórica y práctica frente a las variables analizadas, la enseñanza, el aprendizaje y el teatro; no obstante, se identifican conocimientos más estructurados sobre la disciplina que sobre las otras dos cuestiones indagadas.

Palabras clave: concepciones, educación artística, formación docente, teatro, enseñanza teatral, aprendizaje teatral.

\begin{abstract}
This research revolves around the concepts of teaching, learning and theater in three teachers of the Bachelor of Performing Arts at the Antonio Nariño University located in Bogotá, Colombia. Given that there are few investigations on the subject with teachers of this discipline, the need arises to deepen and build new references that contribute to this field of study. In this sense, an analysis is made of the conceptions and practices of the teachers involved. The research is qualitative and the data treatment is carried out by means of the frequency analysis produced by the coding carried out. It is concluded that a theoretical and practical wealth is evidenced in front of the analyzed variables, teaching, learning and theater, however, more structured knowledge on the discipline is identified than on the other two questions investigated.
\end{abstract}

Key words: Conceptions, Artistic Education, Teacher Training, Theatre, Theatrical Teaching, Theatrical Learning.

\title{
Introducción
}

En diversas investigaciones (Orozco, 2013; Bertoldi, Marano y Rodríguez, 2014) se observa que, en la mayoría de los casos, es posible encontrar un conflicto ideológico entre el arte como disciplina y la pedagogía en función de la educación artística, por lo que se han generado diversas discusiones en el ámbito educativo y es casi imposible vislumbrar los discursos y objetivos de los docentes artistas.

Lo anterior ha provocado que se haya instalado una tradición en las aulas universitarias sobre lo que enseñan estos docentes, que se basa en contenidos propios de su formación actoral, y que en ocasiones deja de lado los aspectos pedagógicos. Por eso, el problema es mayor cuando Alfonso (2012) indica que "los formadores que en su mayoría son artistas en ejercicio, han sido formados en escuelas no formales de teatro" (p. 4).

Al parecer, esto no es un evento aislado de la educación artística, y específicamente del teatro, pues, como se mencionó anteriormente, en otras áreas del conocimiento también se presenta dicha situación, como lo indican Briceño (2013) y Barbosa (2016) en el caso de los profesores universitarios de ciencia, en el cual predomina el interés por lo disciplinar en lugar de los aspectos pedagógicos.

Respecto a las principales problemáticas del profesor de teatro universitario, Alfonso y Rickenmann (2012) afirman que los maestros de teatro no tienen la suficiente formación pedagógica en comparación con la disciplina; por lo tanto, estos maestros son específicamente artistas que poseen una gran riqueza en su saber teatral y, por tal razón, construyen ese conocimiento desde la práctica.

Sumado a lo anterior, se encuentra que hay escasez de investigación a nivel nacional en referencia a la educación artística (Alfonso, 2013); lo que se convierte en otra dificultad, que retrasa futuros avances. Del mismo modo, este 
autor expresa que existen vacíos conceptuales respecto a los procesos de enseñanza y aprendizaje, gracias al mismo problema; por lo tanto, estos formadores transmiten conocimientos desde los contenidos propios de la formación teatral, en un espacio donde se necesita más que estos saberes propios.

Paralelamente, Briceño (2013) advierte que en disciplinas como las ciencias este tipo de situaciones se convierte en un problema, porque las concepciones inadecuadas sobre el aprendizaje y la enseñanza pueden conllevar al desarrollo de prácticas tradicionales. En este sentido, se puede afectar el proceso educativo de los maestros artistas en formación al momento de abordar aspectos disciplinares que no tienen un componente pedagógico.

Teniendo en cuenta lo expuesto, se analizan las concepciones de tres maestros de teatro de la licenciatura en cuestión frente a lo disciplinar y lo pedagógico, como lo señala Alfonso (2012), ahondando propiamente en las concepciones sobre el teatro, la enseñanza, el aprendizaje y, a su vez, contrastando dichos conocimientos (concepciones) con una observación de las prácticas de los docentes que participan en esta investigación.

Así, se hace indispensable fortalecer los conocimientos de los docentes de educación artística desde las perspectivas mencionadas en las problemáticas para mejorar sus prácticas pedagógicas. Por lo tanto, es significativo tener en cuenta sus experiencias pedagógicas para construir un conocimiento propio del saber (Bertoldi, Marano y Rodríguez, 2014) y sacar partido de su potencial en cuanto a lo pedagógico y, así mismo, permitirles ser conscientes de la importancia de la educación teatral, para que lleguen a ser parte de ella (Vieites, 2014, 2015).

El beneficio por determinar estas metodologías cobra importancia para los futuros docentes para que puedan entender, reflexionar y apropiar sus conocimientos, no solo a nivel disciplinar sino también a nivel pedagógico (Orce y Mare, 2016).

\section{Metodología}

La investigación es cualitativa pero el análisis es estadístico, y se realiza con el programa ATLAS ti 7.5, el cual permitió organizar los códigos y frecuencias de cada uno de los casos analizados (descritos más adelante). La siguiente tabla ejemplifica las fuentes revisadas de donde se consiguieron los datos.

Se debe tener en cuenta la nomenclatura del Atlas ti. 7.5 para ubicar las citas dentro del listado de las fuentes de datos de la tabla 1 . Ejemplo: P1: 6 (21:25) significa P 1: $6=$ cita 6 del documento 1 (tabla 1); 21:23 = inicio de la cita en el párrafo 21, finaliza la cita en el párrafo 23 del documento $\mathrm{P}$.
Tabla 1. Lista de datos analizados

\begin{tabular}{|c|l|}
\hline Documentos & \multicolumn{1}{|c|}{ Fuente de datos } \\
\hline P1 & Entrevista al profesor CAC \\
\hline P2 & Observación 1 (sexto semestre) \\
\hline P3 & Observación 2 (sexto semestre) \\
\hline P4 & $\begin{array}{l}\text { Contenido programático } \\
\text { (Teatro de la antigüedad) }\end{array}$ \\
\hline P5 & Entrevista a la profesora PAS \\
\hline P6 & Observación 1 (tercer semestre) \\
\hline P7 & Observación 2 (tercer semestre) \\
\hline P8 & Contenido programático (Teatro \\
\hline P9 & Latinoamericano) \\
\hline P10 & Ontrevista al profesor MIY \\
\hline P11 & Observación 1 (primer semestre) \\
\hline P12 & Contenido programático \\
\hline
\end{tabular}

Fuente: elaboración propia 


\section{Participantes}

Se seleccionaron como muestra tres profesores del programa, identificados en esta investigación con los siguientes seudónimos: PAS, CAC y MIY. Respecto a su formación inicial se puede decir que los tres son licenciados, uno de ellos empezó su carrera artística desde muy joven; adicionalmente, han tenido estudios posgraudales. Actualmente, dos de ellos continúan formándose en áreas del conocimiento disciplinar. Los profesores fueron escogidos de acuerdo con su experiencia como docentes de teatro del programa.

\section{Recolección de datos: entrevistas, observaciones y contenido prográmtico}

Para el diseño de las entrevistas se pensó en algunas inquietudes surgidas en este trabajo, como también en algunas preguntas propuestas en estudios realizados por el doctor Miguel Alfonso (2013b) de la UPN, además de las inquietudes propias del autor. Las preguntas realizadas fueron las siguientes: ¿el teatro qué es para usted? ¿Cómo lo define? ¿Lo considera una disciplina? ¿Qué entiende por enseñanza del teatro? ¿Cómo la enseña? Explique cuál es la metodología que usa para enseñar el teatro. ¿Cómo ha sido el proceso de enseñar el teatro en la licenciatura? ¿Cómo aporta a sus estudiantes para que desarrollen su práctica pedagógica en lo teatral? ¿Cuál es la particularidad de su labor? ¿Qué entiende por aprendizaje? ¿Cómo logra que sus estudiantes aprendan más? ¿Qué estrategias ha realizado que le ayudan a que los estudiantes aprendan? Con más exactitud: ¿qué metas de aprendizajes se persiguen en teatro? ¿Cómo verifica que sus estudiantes tengan prácticas apropiadas de enseñanza del teatro en las instituciones donde hacen sus prácticas? ¿Qué cree usted que los estudiantes se llevan de sus clases? ¿Qué relación cree que debe existir entre el maestro y el estudiante para el desarrollo de las prácticas pedagógicas?

Las entrevistas se realizaron de manera individual y los docentes fueron informados anteriormente del procedimiento, el cual se desarrolló dentro de las instalaciones del programa.

La duración de cada entrevista fue de aproximadamente una hora. Para las observaciones, estas se grabaron en audio y video y fueron transcritas para ser analizadas con Atlas Tic 7.5 , al igual que los contenidos programáticos y las entrevistas con el siguiente sistema de códigos.

\section{Sistema de categorías y códigos para el análisis de las concepciones}

Se construye un sistema de códigos y categorías que emergen del marco teórico y otros que emergen de la experiencia de los autores en la enseñanza y aprendizaje del teatro. La siguiente tabla presenta el análisis de las concepciones de los docentes del programa, cuyas fuentes de datos salen de las entrevistas realizadas y los contenidos programáticos que los mismos docentes elaboran al iniciar el periodo académico.

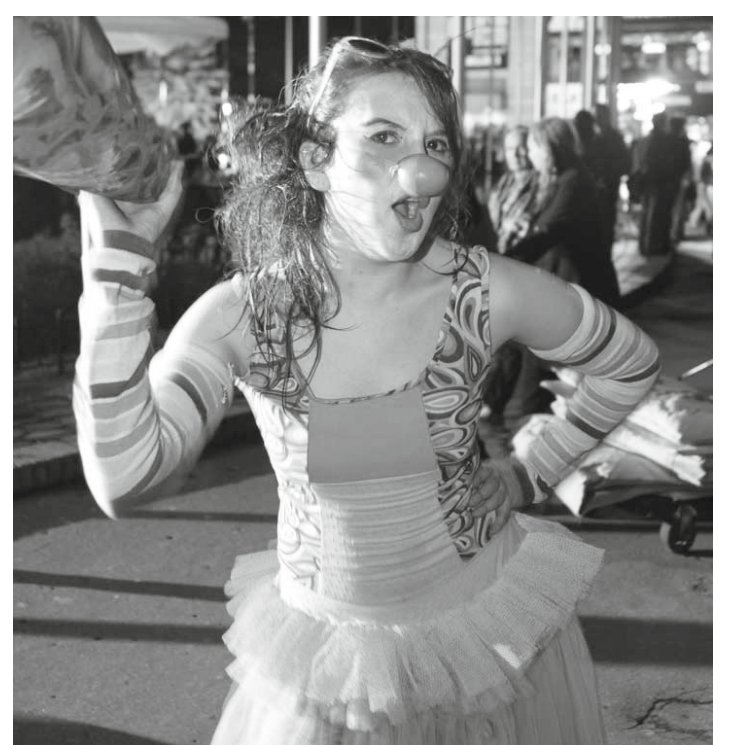


Tabla 2. Sistema de categorías y códigos de análisis de la información desde las entrevistas y el contenido programático

\begin{tabular}{|c|c|c|}
\hline \multicolumn{3}{|c|}{ Análisis de las concepciones de los docentes } \\
\hline Códigos & Categorías & Definición \\
\hline CDT & \multicolumn{2}{|c|}{ Concepción sobre el teatro } \\
\hline CDT-EV & $\begin{array}{l}\text { El teatro como forma } \\
\text { de vida }\end{array}$ & El teatro como su profesión y fuente principal de ingresos. \\
\hline CDT-GA & $\begin{array}{l}\text { El teatro como guía de } \\
\text { aprendizaje }\end{array}$ & $\begin{array}{l}\text { Por medio del teatro se aprende, entiende, comprende y explora } \\
\text { la humanidad. }\end{array}$ \\
\hline CDT-TD & $\begin{array}{l}\text { Disciplina como } \\
\text { conjunto de reglas }\end{array}$ & $\begin{array}{l}\text { El teatro es una disciplina sobre el entrenamiento, la responsa- } \\
\text { bilidad y el cumplimiento de sus normas. }\end{array}$ \\
\hline CDT-DI & Teatro como disciplina & $\begin{array}{l}\text { Reconoce el teatro como un campo de estudio que se encuentra } \\
\text { dentro del arte. }\end{array}$ \\
\hline CDT-TE & El teatro desde la ética & $\begin{array}{l}\text { El ejercicio teatral se debe hacer de manera ética o no tendría } \\
\text { sentido y tendería a morir. }\end{array}$ \\
\hline CET & \multicolumn{2}{|c|}{ Concepciones sobre la enseñanza } \\
\hline CET-CM & $\begin{array}{l}\text { Profesor centrado en sí } \\
\text { mismo }\end{array}$ & $\begin{array}{l}\text { Kugel (1993) dice que se "caracteriza por explicar demasiado } \\
\text { rápido, con poca claridad, no saber distribuir los contenidos en } \\
\text { el tiempo, temor a que les sobre tiempo, temor a las preguntas } \\
\text { de los alumnos". }\end{array}$ \\
\hline CET-EB & Enseñanza bilateral & $\begin{array}{l}\text { Según Jaramillo (2013), es aquella dónde el estudiante y el } \\
\text { docente participan por igual en clase. }\end{array}$ \\
\hline CET-PR & $\begin{array}{l}\text { Principios de la } \\
\text { enseñanza }\end{array}$ & $\begin{array}{l}\text { Para la enseñanza del teatro se parte de acuerdos, principios y } \\
\text { normas establecidos entre los estudiantes y el docente. }\end{array}$ \\
\hline $\mathrm{CET}-\mathrm{CH}$ & $\begin{array}{l}\text { El contenido } \\
\text { programático como } \\
\text { herramienta }\end{array}$ & $\begin{array}{l}\text { Se refiere a que se enseña con base al contenido programático } \\
\text { que se trabaja en el programa (Alfonso, 2012). }\end{array}$ \\
\hline CET-ET & $\begin{array}{l}\text { Enseñanza a partir de } \\
\text { la teoría }\end{array}$ & $\begin{array}{l}\text { Se refiere a que el estudiante y el docente deben actualizarse } \\
\text { constantemente con textos, lecturas dramáticas, textos educa- } \\
\text { tivos, ayudas audiovisuales como el teatro, la televisión y el cine } \\
\text { (Bertoldi, Marano y Rodríguez, 2014). }\end{array}$ \\
\hline CET-EP & $\begin{array}{l}\text { Enseñanza a partir de } \\
\text { la práctica }\end{array}$ & $\begin{array}{l}\text { Se refiere a que el estudiante debe estar en constante práctica } \\
\text { con lo aprendido, ensayando y presentando ejercicios teatrales } \\
\text { (Dip y Gómez, 2014). }\end{array}$ \\
\hline CET-CD & $\begin{array}{l}\text { Capacitación del } \\
\text { docente }\end{array}$ & $\begin{array}{l}\text { Se refiere a la actualización permanente por medio de cursos, } \\
\text { talleres, seminarios, entre otros. }\end{array}$ \\
\hline CET-AE & $\begin{array}{l}\text { Autonomía en la } \\
\text { enseñanza }\end{array}$ & $\begin{array}{l}\text { Se rescata el valor de las distintas maneras de enseñar el teatro } \\
\text { en los docentes de la licenciatura. }\end{array}$ \\
\hline CET-EL & Enseñar un legado & $\begin{array}{l}\text { Preocupación por mantener vivo un legado adquirido como } \\
\text { egresado del programa; por lo tanto, enseña lo que aprendió y } \\
\text { cómo lo aprendió. }\end{array}$ \\
\hline CET-EN & Enseñanza por niveles & $\begin{array}{l}\text { Corresponde a los distintos procesos de enseñanza en los dis- } \\
\text { tintos semestres y que se da mejor en unos niveles a diferencia } \\
\text { de otros. }\end{array}$ \\
\hline CET-EM & $\begin{array}{l}\text { Enseñanza } \\
\text { multidisciplinar }\end{array}$ & $\begin{array}{l}\text { Se refiere a que en el teatro se debe acceder, profundizar o nutrir } \\
\text { necesariamente de otras disciplinas teatrales o de expresión } \\
\text { corporal, y viceversa (Flores, 2013). }\end{array}$ \\
\hline
\end{tabular}




\begin{tabular}{|c|c|c|}
\hline \multicolumn{3}{|c|}{ Análisis de las concepciones de los docentes } \\
\hline Códigos & Categorías & Definición \\
\hline CET-ES & Enseñanza específica & $\begin{array}{l}\text { Es la preocupación por enseñar un tema en específico del teatro } \\
\text { (por ejemplo, voz, cuerpo, texto, etc.). }\end{array}$ \\
\hline CET-EE & Ejemplos de enseñanza & $\begin{array}{l}\text { Recurre a las anécdotas sobre la práctica pedagógica que le } \\
\text { enseñaron a mejorar sus prácticas. }\end{array}$ \\
\hline CET-RE & Rol educativo & $\begin{array}{l}\text { Se refiere a saber diferenciar las prácticas de enseñanza del } \\
\text { teatro, la dirección, dramaturgia entre otros roles (Alfonso, } \\
\text { 2013). }\end{array}$ \\
\hline CET-DT & Didáctica teatral & $\begin{array}{l}\text { Son las metodologías propias del teatro utilizadas para la crea- } \\
\text { ción o aprendizaje propio de la disciplina (Alfonso, 2013). }\end{array}$ \\
\hline CAP & \multicolumn{2}{|c|}{ Concepciones sobre el aprendizaje } \\
\hline CAP-CA & $\begin{array}{l}\text { Profesor centrado en el } \\
\text { alumno }\end{array}$ & $\begin{array}{l}\text { Kugel (1993) dice que "el docente comienza a centrar sus pen- } \\
\text { samientos en sus estudiantes y en cómo hacer que ellos puedan } \\
\text { retener más y mejor lo que les está enseñando". }\end{array}$ \\
\hline CAP-IP & $\begin{array}{l}\text { Intercambio } \\
\text { permanente }\end{array}$ & $\begin{array}{l}\text { Se refiere al intercambio de saberes o conocimiento que brinda } \\
\text { el medio ambiente, la sociedad y uno mismo. }\end{array}$ \\
\hline CAP-AP & $\begin{array}{l}\text { Aprendizaje para la } \\
\text { práctica docente }\end{array}$ & $\begin{array}{l}\text { Todo lo que los estudiantes o compañeros de trabajo pueden } \\
\text { brindar al docente para mejorar sus prácticas educativas. }\end{array}$ \\
\hline CAP-IC & $\begin{array}{l}\text { Aprendizaje por } \\
\text { intuición o casualidad }\end{array}$ & $\begin{array}{l}\text { Son los hechos que se dan en la práctica pedagógica y teatral } \\
\text { que se manejan por la intuición o el azar de los docentes y estu- } \\
\text { diantes (Alfonso, 2013). }\end{array}$ \\
\hline CAP-PR & $\begin{array}{l}\text { Aprendizaje } \\
\text { predispuesto }\end{array}$ & $\begin{array}{l}\text { Significa que los estudiantes vienen predispuestos a aprender } \\
\text { de un docente en específico. }\end{array}$ \\
\hline CAP-OC & $\begin{array}{l}\text { Organización } \\
\text { curricular }\end{array}$ & $\begin{array}{l}\text { Se piensa que existen problemas en la propuesta curricular } \\
\text { abordada en el programa (Dimatteo, 2016). }\end{array}$ \\
\hline CAP-AR & Aprendizaje reflexivo & $\begin{array}{l}\text { A partir de un foro al finalizar cada clase se habla de aspectos } \\
\text { positivos y negativos de lo sucedido en clase para mejorar el } \\
\text { proceso. }\end{array}$ \\
\hline CAP-MO & $\begin{array}{l}\text { Aprendizaje por } \\
\text { motivación }\end{array}$ & $\begin{array}{l}\text { Se refiere a las metodologías innovadoras y motivantes que el } \\
\text { docente busca para que el estudiante aprenda. }\end{array}$ \\
\hline CAP-LA & Lugar de aprendizaje & $\begin{array}{l}\text { Se refiere a que es importante cambiar de espacios donde } \\
\text { sucede la enseñanza y el aprendizaje para un mejor desarrollo. }\end{array}$ \\
\hline CAP-DA & $\begin{array}{l}\text { Deserción del } \\
\text { aprendizaje }\end{array}$ & $\begin{array}{l}\text { Son las situaciones donde los docentes tienen dificultades en } \\
\text { los procesos de enseñanza y aprendizaje del teatro y terminan } \\
\text { abandonando el proceso de alguna manera (Bertoldi, Marano, } \\
\text { y Rodríguez, 2014). }\end{array}$ \\
\hline CEA & \multicolumn{2}{|c|}{ Concepciones de la relación enseñanza y aprendizaje } \\
\hline CEA-RV & $\begin{array}{l}\text { Relación basada en } \\
\text { valores }\end{array}$ & $\begin{array}{l}\text { Son aquellos valores que se deben tener en cuenta en los pro- } \\
\text { cesos de enseñanza y aprendizaje entre docentes y estudiantes. }\end{array}$ \\
\hline CEA-FP & Fraternizar prácticas & $\begin{array}{l}\text { Los estudiantes ponen en práctica sus conocimientos académi- } \\
\text { cos o profesionales con sus compañeros por medio de ejercicios } \\
\text { (Bertoldi, Marano y Rodríguez, 2014). }\end{array}$ \\
\hline CEA-PA & $\begin{array}{l}\text { La enseñanza como } \\
\text { proceso de aprendizaje }\end{array}$ & $\begin{array}{l}\text { Asume el rol de enseñanza como de aprendizaje al mismo } \\
\text { tiempo (Bertoldi, 2014). }\end{array}$ \\
\hline
\end{tabular}

Fuente: entrevistas y contenidos programáticos 
Tabla 3. Sistema de categorías y códigos de análisis de la información desde las observaciones

\begin{tabular}{|c|c|c|}
\hline \multicolumn{3}{|c|}{ Análisis de las prácticas de los docentes } \\
\hline Códigos & Categorías & Definición \\
\hline CET & \multicolumn{2}{|c|}{ Concepciones de la práctica sobre la enseñanza } \\
\hline CET-CM & $\begin{array}{l}\text { Profesor centrado en sí } \\
\text { mismo }\end{array}$ & $\begin{array}{l}\text { Kugel (1993) dice que se "caracteriza por explicar demasiado } \\
\text { rápido, con poca claridad, no saber distribuir los contenidos en } \\
\text { el tiempo, temor a que les sobre tiempo, temor a las preguntas } \\
\text { de los alumnos". }\end{array}$ \\
\hline CET-DM & $\begin{array}{l}\text { Profesor centrado en el } \\
\text { dominio de la materia }\end{array}$ & $\begin{array}{l}\text { Kugel (1993) dice que "el profesorado alcanza un mayor } \\
\text { dominio de las materias que imparte y su preocupación por } \\
\text { quedarse sin palabras pasa a convertirse en preocupación por } \\
\text { no tener tiempo para transmitirlo todo". }\end{array}$ \\
\hline CET-PR & $\begin{array}{l}\text { Principios de la } \\
\text { enseñanza }\end{array}$ & $\begin{array}{l}\text { Para la enseñanza del teatro se parte de acuerdos, principios y } \\
\text { normas establecidos entre los estudiantes y el docente. }\end{array}$ \\
\hline CET-DT & Didáctica teatral & $\begin{array}{l}\text { Son las metodologías propias del teatro utilizadas para la } \\
\text { creación o aprendizaje propio de la disciplina (Alfonso y } \\
\text { Rickenmann, 2012). }\end{array}$ \\
\hline CET-LT & Lenguaje teatral & $\begin{array}{l}\text { Alfonso y Rickenmann (2012) se refieren al lenguaje usado que } \\
\text { es un poco más coloquial y que usa conceptos propiamente } \\
\text { disciplinares. }\end{array}$ \\
\hline CET-LP & Lenguaje pedagógico & $\begin{array}{l}\text { Alfonso y Rickenmann (2012) se refieren al lenguaje usado que } \\
\text { es un poco más técnico y los conceptos son más pedagógicos. }\end{array}$ \\
\hline PARE & $\begin{array}{l}\text { Participación activa del } \\
\text { estudiante }\end{array}$ & $\begin{array}{l}\text { Se refiere a los momentos de la clase donde el estudiante parti- } \\
\text { cipa activamente (Briceño, 2013). }\end{array}$ \\
\hline PARE-R & $\begin{array}{l}\text { Participación activa y } \\
\text { razonada del estudiante }\end{array}$ & $\begin{array}{l}\text { Se refiere a los momentos de la clase en que el estudiante } \\
\text { participa activamente y sus respuestas son razonadas desde lo } \\
\text { aprendido. }\end{array}$ \\
\hline PDO-IN & $\begin{array}{l}\text { Indicaciones del } \\
\text { docente }\end{array}$ & $\begin{array}{l}\text { Se refiere a que el docente participa activamente desde las } \\
\text { indicaciones a nivel de dirección teatral. }\end{array}$ \\
\hline PP-DOC & $\begin{array}{l}\text { Promoción y } \\
\text { participación del } \\
\text { docente }\end{array}$ & $\begin{array}{l}\text { Son los momentos en que el docente, aparte de dar las } \\
\text { indicaciones, explica por qué y para qué da esas indicaciones. }\end{array}$ \\
\hline CET-DIR & Enseñanza dirigida & $\begin{array}{l}\text { Se refiere a la enseñanza que se da por medio de la dirección } \\
\text { teatral. }\end{array}$ \\
\hline CET-EE & Ejemplos de enseñanza & $\begin{array}{l}\text { Recurre a las anécdotas sobre la práctica pedagógica que le } \\
\text { enseñaron a mejorar sus prácticas (Alfonso, 2012). }\end{array}$ \\
\hline CAP & Concepciones de la prác & tica sobre el aprendizaje \\
\hline CAP-CA & $\begin{array}{l}\text { Profesor centrado en el } \\
\text { alumno }\end{array}$ & $\begin{array}{l}\text { Kugel (1993) dice que "el docente comienza a centrar sus pen- } \\
\text { samientos en sus estudiantes y en cómo hacer que ellos puedan } \\
\text { retener más y mejor lo que les está enseñando". }\end{array}$ \\
\hline
\end{tabular}

Fuentes de datos: observaciones 


\section{Resultados y análisis}

A continuación, se presenta la tabla 4 que ejemplifica el total de las frecuencias obtenidas de los códigos relacionados con las citas, que muestra un total de 888; y en los códigos empleados se encuentran un total de 41 , los cuales se explicaron de manera minuciosa anteriormente.

En la tabla 4 se observan los códigos obtenidos de la observación que tienen una frecuencia bastante alta y que corresponden a: código PARE definido en la tabla de análisis de la práctica, como la participación activa del estudiante. También se encuentra el código
PP-DOC, definido como la promoción y participación del docente, que nos da a entender principalmente que la enseñanza del teatro en el programa se realiza de manera bilateral, es decir, que el docente y los estudiantes participan de forma equilibrada en las clases como menciona (Jaramillo, 2013) en la tabla de análisis de concepciones de la enseñanza.

Posteriormente, se presenta el análisis de frecuencias de las concepciones de enseñanza, aprendizaje, teatro y su relación, en las que se analizarán de manera individualizada las concepciones predominantes en cada caso.

Tabla 4. Matriz general de resultados

\begin{tabular}{|c|c|c|c|c|c|c|c|c|c|c|c|c|c|c|}
\hline & \multicolumn{12}{|c|}{ Fuentes de datos } & \multirow{3}{*}{$\begin{array}{c}\text { Profesores } \\
\text { Totales }\end{array}$} \\
\hline & & \multicolumn{4}{|c|}{ CAC } & \multicolumn{4}{|c|}{ PAS } & \multicolumn{4}{|c|}{ MIY } & \\
\hline & & P1 & P2 & P3 & P4 & P5 & P6 & P7 & P8 & P9 & P10 & P11 & P13 & \\
\hline \multirow{20}{*}{ 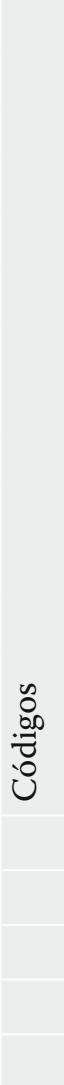 } & CAP-AP & 1 & 0 & 0 & 0 & 2 & 0 & 0 & 0 & 0 & 0 & 0 & 0 & 3 \\
\hline & CAP-AR & 4 & 0 & 0 & 1 & 0 & 0 & 0 & 1 & 1 & 0 & 0 & 0 & 7 \\
\hline & CAP-CA & 10 & 0 & 2 & 2 & 6 & 0 & 0 & 2 & 3 & 1 & 0 & 1 & 27 \\
\hline & CAP-DA & 1 & 0 & 0 & 0 & 1 & 0 & 0 & 0 & 0 & 0 & 0 & 0 & 2 \\
\hline & CAP-IC & 1 & 0 & 0 & 0 & 2 & 0 & 0 & 0 & 0 & 0 & 0 & 0 & 3 \\
\hline & CAP-IP & 0 & 0 & 0 & 0 & 1 & 0 & 0 & 0 & 1 & 0 & 0 & 0 & 2 \\
\hline & CAP-LA & 0 & 0 & 0 & 0 & 0 & 0 & 0 & 0 & 1 & 0 & 0 & 1 & 2 \\
\hline & CAP-MO & 0 & 0 & 0 & 0 & 0 & 0 & 0 & 0 & 2 & 0 & 0 & 0 & 2 \\
\hline & CAP-OC & 0 & 0 & 0 & 0 & 1 & 0 & 0 & 0 & 0 & 0 & 0 & 0 & 1 \\
\hline & CAP-PR & 1 & 0 & 0 & 0 & 1 & 0 & 0 & 0 & 0 & 0 & 0 & 0 & 2 \\
\hline & CDT-DI & 0 & 0 & 0 & 0 & 1 & 0 & 0 & 0 & 0 & 0 & 0 & 0 & 1 \\
\hline & CDT-EV & 1 & 0 & 0 & 0 & 0 & 0 & 0 & 0 & 1 & 0 & 0 & 0 & 2 \\
\hline & CDT-GA & 1 & 0 & 0 & 0 & 1 & 0 & 0 & 0 & 1 & 0 & 0 & 0 & 3 \\
\hline & CDT-TD & 1 & 0 & 0 & 0 & 0 & 0 & 0 & 0 & 1 & 0 & 0 & 0 & 2 \\
\hline & CDT-TE & 1 & 0 & 0 & 0 & 0 & 0 & 0 & 0 & 0 & 0 & 0 & 0 & 1 \\
\hline & CEA-FP & 1 & 0 & 0 & 0 & 0 & 0 & 0 & 0 & 0 & 0 & 0 & 0 & 1 \\
\hline & CEA-PA & 1 & 0 & 0 & 0 & 1 & 0 & 0 & 0 & 0 & 0 & 0 & 0 & 2 \\
\hline & CEA-RV & 2 & 0 & 0 & 0 & 3 & 0 & 0 & 0 & 1 & 0 & 0 & 0 & 6 \\
\hline & CET- EL & 0 & 0 & 0 & 0 & 0 & 0 & 0 & 0 & 1 & 0 & 0 & 0 & 1 \\
\hline & CET-AE & 1 & 0 & 0 & 0 & 0 & 0 & 0 & 0 & 0 & 0 & 0 & 0 & 1 \\
\hline
\end{tabular}




\begin{tabular}{|c|c|c|c|c|c|c|c|c|c|c|c|c|c|}
\hline & \multicolumn{12}{|c|}{ Fuentes de datos } & \multirow{3}{*}{$\begin{array}{c}\text { Profesores } \\
\text { Totales }\end{array}$} \\
\hline & \multicolumn{4}{|c|}{ CAC } & \multicolumn{4}{|c|}{ PAS } & \multicolumn{4}{|c|}{ MIY } & \\
\hline & P1 & P2 & P3 & P4 & P5 & P6 & P7 & P8 & P9 & P10 & P11 & P13 & \\
\hline CET-CD & 3 & 0 & 0 & 0 & 0 & 0 & 0 & 0 & 1 & 0 & 0 & 0 & 4 \\
\hline CET-CH & 3 & 0 & 0 & 1 & 0 & 0 & 0 & 1 & 0 & 0 & 0 & 1 & 6 \\
\hline CET-CM & 0 & 0 & 0 & 0 & 0 & 1 & 3 & 0 & 0 & 0 & 0 & 0 & 4 \\
\hline CET-DIR & 1 & 61 & 5 & 0 & 1 & 3 & 8 & 0 & 0 & 2 & 0 & 0 & 81 \\
\hline CET-DM & 0 & 1 & 0 & 0 & 0 & 0 & 0 & 0 & 0 & 1 & 0 & 0 & 2 \\
\hline CET-DT & 4 & 0 & 0 & 1 & 0 & 5 & 0 & 1 & 4 & 0 & 0 & 1 & 16 \\
\hline CET-EB & 3 & 0 & 0 & 0 & 0 & 0 & 0 & 0 & 0 & 0 & 0 & 0 & 3 \\
\hline CET-EE & 0 & 1 & 1 & 0 & 2 & 0 & 0 & 0 & 0 & 0 & 0 & 0 & 4 \\
\hline CET-EM & 0 & 0 & 0 & 2 & 3 & 0 & 0 & 0 & 1 & 0 & 0 & 1 & 6 \\
\hline CET-EN & 0 & 0 & 0 & 0 & 1 & 0 & 0 & 0 & 0 & 0 & 0 & 0 & 1 \\
\hline CET-EP & 3 & 0 & 0 & 1 & 0 & 0 & 0 & 2 & 0 & 0 & 0 & 1 & 7 \\
\hline CET-ES & 1 & 0 & 0 & 0 & 0 & 0 & 0 & 0 & 3 & 0 & 0 & 0 & 4 \\
\hline CET-ET & 5 & 0 & 0 & 3 & 0 & 0 & 0 & 1 & 2 & 0 & 1 & 1 & 13 \\
\hline CET-LP & 0 & 0 & 0 & 0 & 0 & 0 & 1 & 0 & 0 & 2 & 0 & 0 & 3 \\
\hline CET-LT & 0 & 11 & 10 & 0 & 0 & 1 & 4 & 0 & 0 & 1 & 0 & 0 & 27 \\
\hline CET-PR & 3 & 0 & 0 & 1 & 1 & 0 & 1 & 1 & 0 & 0 & 0 & 1 & 8 \\
\hline CET-RE & 3 & 0 & 0 & 0 & 7 & 0 & 0 & 0 & 1 & 0 & 0 & 0 & 11 \\
\hline PARE & 0 & 101 & 60 & 0 & 0 & 11 & 44 & 0 & 0 & 16 & 28 & 0 & 260 \\
\hline PARE-R & 0 & 15 & 7 & 0 & 0 & 0 & 22 & 0 & 0 & 5 & 17 & 0 & 66 \\
\hline PDO-IN & 0 & 19 & 0 & 0 & 0 & 3 & 0 & 0 & 0 & 9 & 0 & 0 & 31 \\
\hline PP-DOC & 0 & 20 & 77 & 0 & 0 & 16 & 49 & 0 & 0 & 25 & 67 & 0 & 254 \\
\hline Total & 56 & 229 & 162 & 14 & 35 & 40 & 132 & 10 & 25 & 62 & 113 & 10 & 883 \\
\hline
\end{tabular}

Fuente: elaboración propia

\section{Relación de concepciones y práctica del profesor CAC}

En la figura 1 se presentan los códigos encontrados en las concepciones y en la práctica del docente en su práctica pedagógica.

En resumen, este gráfico corresponde a algunas citas, tales como: "yo tengo que entender que el estudiante no necesariamente debe tener la respuesta inmediata, pues porque está aprendiendo aquí, está formándose aquí, está buscando sus preguntas, entonces yo tengo que ayudarles a buscar esas preguntas" (P1: $1: 63$ [48:48]).
Figura 1. Relación entre las concepciones y la práctica del docente CAC

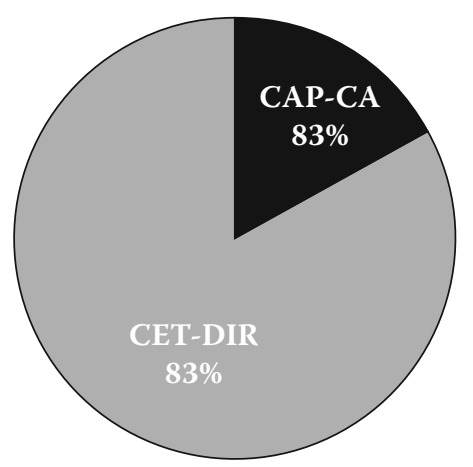

Fuente: elaboración propia 
El CAP-CA evidenciado anteriormente es el código más repetitivo del profesor CAC, debido a que él demuestra cierta preocupación por sus estudiantes respecto a que ellos logren mejorar su aprendizaje; por lo tanto, se vale de las estrategias que cree más apropiadas para el desarrollo de sus clases, tal y como lo indica el Dr. Kugel en la investigación de Briceño (2013). En el caso del profesor CAC se evidencia cuando, en clase, les dice a sus estudiantes: "Porque lo que podríamos trabajar, es que pongan eso atrás al otro lado y la cerca al otro lado. Pónganlo en perspectiva; haber, llenen esa obra de perspectiva (P2: 2:136 [146:146])". Y agrega: "Te veo, te veo a ti y digo esta bonita la china, pero no la quiero ver bonita, te quiero ver inocente" (P3: 3:177 [4:4]).

Posteriormente, refiriéndonos a las concepciones de la enseñanza en el código CET-DIR, que tiene un número considerablemente alto de frecuencias, se encuentra que el profesor CAC pretende mostrar a sus estudiantes que se puede usar la dirección teatral como método o estrategia de enseñanza del teatro en los estudiantes.

Encontramos en las concepciones de enseñanza el código CET-DIR que tiene una frecuencia de 67, la cual nos aclara que el docente enseña por medio de la dirección teatral; y en las concepciones del aprendizaje, el código CAP-CA, con una frecuencia de 14, que nos deja ver el interés y la preocupación que tiene para que sus estudiantes aprendan la materia lo mejor posible; por lo tanto, se puede observar claramente que en estos dos códigos hay una relación entre lo que dice el profesor y lo que hace en sus clases.

\section{Relación de las concepciones y la práctica de la profesora PAS}

El siguiente gráfico expone los códigos que se identificaron en las concepciones y en las prácticas, en las que se evidencia coherencia entre lo que dice y hace el docente.
Figura 2. Relación de las concepciones y la práctica de la docente PAS

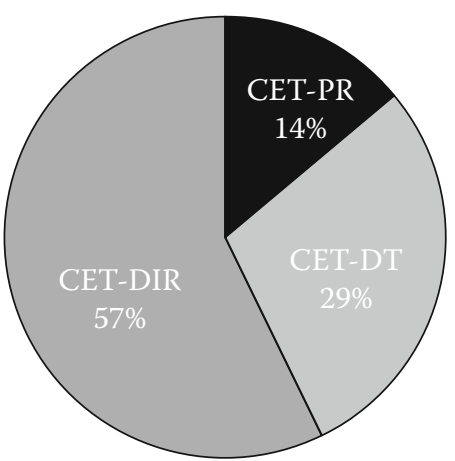

Fuente: elaboración propia

En resumen, este grafico corresponde a algunas citas, tales como: "Hagámonos todos allá, si quieren traer una silla, la que sobrevive de la incandescencia (risas) muy bien, muy bien. Bueno, primer grupo, no, segundo grupo va a leer al primer grupo, ya saben cómo es leer, ¿cierto? Leer es yo vi tal cosa, es describir. ¿Sí? El segundo al primero" (P7: 7:162 [21:21]).

Los ejemplos anteriores nos revelan el código CET-DIR, el cual permite identificar la dirección de un ejercicio teatral y al mismo tiempo un trabajo práctico para que los estudiantes preparen, tal como se evidencia en la siguiente indicación, una actividad teatral: "Nada me enseñaste de este otro mar oculto: la traición. Siguiéndote los pasos, soy transparente" (P6: 6:56 [43:43]). Y agrega: "Los negros han malinterpretado las generosas leyes de la manumisión. Confundiendo libertad con libertinaje, ahora se dan a la fuga y abandonan el trabajo para emborracharse" (P6: 6:61 [59:59]).

Estos ejemplos nos muestran el código CETDT, en el cual se ve la didáctica que usa la docente para enseñar a través de la lectura, para despertar la imaginación y la creatividad en los estudiantes: "Yo trazo como unas rutas de aprendizaje que negocio con ellos y, digamos, que en proceso pueden ir cambiando con el nivel de desarrollo que se va dando" (P5: 5:43 [47:47]). A lo que la profesora PAS agrega:

Revista PAPELES • ISSN 0123-0670 • Vol. 12(23) • pp. 70-85 • Enero-junio de 2020 
"Los acuerdos iniciales con los estudiantes son fundamentales para el éxito escolar" (P8: 8:9 [204:204]).

El código CET-PR nos permite apreciar la importancia y el orden que le da la docente a la asignatura, ya que parte de acuerdos que pueden ser flexibles para sus estudiantes durante el periodo académico.

El código CET-DIR muestra una frecuencia de 12 como la más alta, la cual da a entender que la docente usa la dirección teatral o asume el rol de directora para enseñar. El código CET-DT se presenta con una frecuencia de 6 , lo cual muestra que se usa una didáctica particular para sus enseñanzas; y tenemos el código CET-PR, con una frecuencia de 3 , el cual evidencia que para que se dé la enseñanza hay que llegar a unos acuerdos. Se puede observar que, en estos códigos, hay una relación entre lo que dice y hace la profesora en sus clases.

\section{Relación de las concepciones y la práctica del profesor MIY}

En el gráfico que aparece enseguida se observa la relación encontrada entre las entrevistas, contenido programático y observaciones, que dejan en evidencia la coherencia que existe entre lo que dice y hace el docente en sus prácticas pedagógicas.

Figura 3. Relación de las concepciones y la práctica del docente MIY

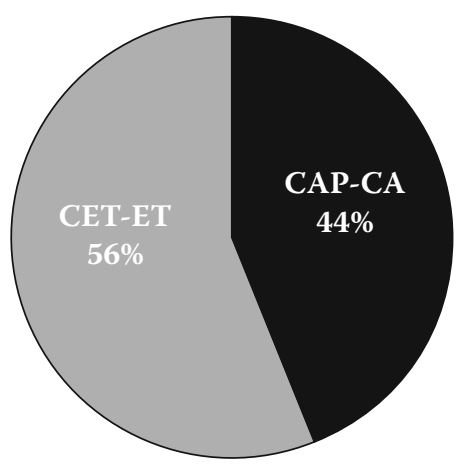

Fuente: elaboración propia
En resumen, este gráfico corresponde a algunas citas del profesor MIY, tales como: "¿Qué libros leyeron? ¿Qué libro tiene que leer?" (P11: 11:96 [185:185]). Y agrega: "La escuela de espectador significa que, en mi materia, por lo menos, se va ocho veces a ver una obra de teatro, es decir, se ven ocho espectáculos mínimo, como alimento para ellos durante este mes" (P9: 9:13 [31:31]). Y finaliza diciendo: "Lectura" (P13: 13:14 [128:128]).

La siguiente cita muestra el código CET-ET, el cual da cuenta de que el docente corrobora el trabajo teórico propuesto a los estudiantes con las tareas dejadas para la casa dentro de la práctica realizada en la clase: "Bueno, yo creo que el principal aporte que yo le hago a los estudiantes es que yo les doy las herramientas para que ellos construyan su conocimiento; aprender a aprender primero y desaprender. En este sentido, creo que mi concepción y los aportes que yo doy es más que despierten una curiosidad" (P9: 9:35 [31:31]). Y concluye diciendo: "Para descubrir, adquirir y apropiarse de las herramientas básicas necesarias en el resto de su carrera y la vida profesional, el estudiante que inicia su ciclo en la formación teatral, solamente a través de la vivencia (praxis) las puede adquirir" (P13: 13: [33:33]).

Los anteriores ejemplos exponen el código CAP-CA, el cual logra evidenciar la preocupación que tiene el docente para que los estudiantes construyan su propio conocimiento, ya que muestra empatía con ellos constantemente. Los códigos relacionados nos dejan ver la coherencia que tiene el docente en cuanto a la importancia que tiene la teoría dentro de sus concepciones y la preocupación para que aprendan la disciplina de la mejor manera.

\section{Análisis de la relación de los profesores CAC, PAS Y MIY}

A continuación, se presentan 3 gráficos que nos muestran los códigos más destacados de los profesores MIY, CAC y PAS conseguidos de los

Universidad Antonio Nariño • Facultad de Ciencias de la Educación 
anteriores análisis. Aquí se pueden observar los códigos CET-DIR, PP-DOC, PARE-R y PARE, que nos muestran la relación entre la enseñanza y el aprendizaje que se da en las clases de teatro en cada uno de los 3 docentes analizados.

Figura 4. Enseñanza y aprendizaje del docente MIY
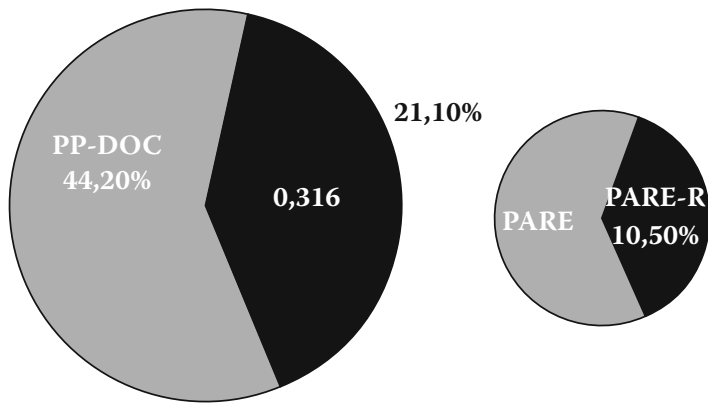

Fuente: elaboración propia

Partiendo de la alta frecuencia del código PP-DOC del docente MIY se puede decir que limita la participación de los estudiantes, como se observa en los códigos PARE y PARE-R, pero muestra que posiblemente es un profesor que tiene dominio de la materia, como se menciona en la investigación de Briceño (2013). En los códigos PARE y PARE-R de los estudiantes del docente MIY se puede observar que tienden a tener un aprendizaje activo, según el mismo autor citado, si se compara con la participación de los estudiantes de los otros docentes, dado que rondan un porcentaje similar.

Figura 5. Enseñanza y aprendizaje del docente CAC

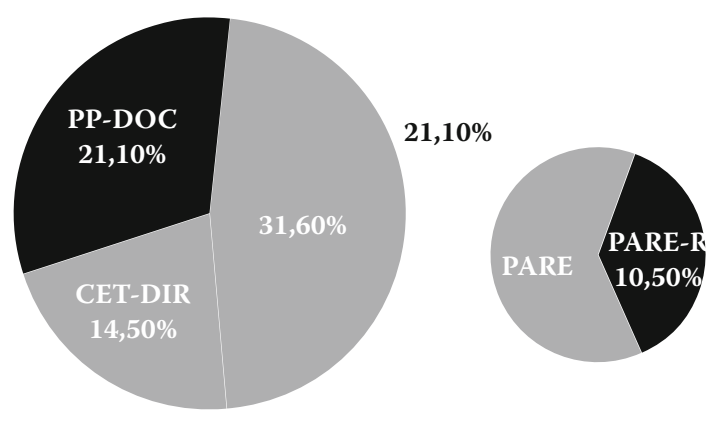

Fuente: elaboración propia
Se puede probar que el docente CAC tiene una participación pedagógica en el código PP-DOC con la mitad de frecuencia del docente MIY, aunque, como se puede observar, si le adicionamos su participación disciplinar, nos da como resultado una aproximación a una participación bilateral en sus clases, lo cual permite que la información y el conocimiento fluyan en el proceso de enseñanza y aprendizaje. Se evidencia a un docente que alterna la teoría y la práctica, plantea problemas, no se preocupa por la cantidad de la enseñanza, sino el cómo lo hace, para que sus estudiantes puedan aprender mejor; sin duda es un profesor preocupado por el alumno, según Briceño (2013). En los estudiantes del docente CAC se evidencia una participación bilateral encaminada a una preocupación centrada por el docente en el estudiante, donde este es visto como sujeto activo, dado que su aprendizaje se da de manera dinámica y logra poner en práctica lo aprendido, como indica el mismo autor.

Figura 6. Enseñanza y aprendizaje de la docente PAS

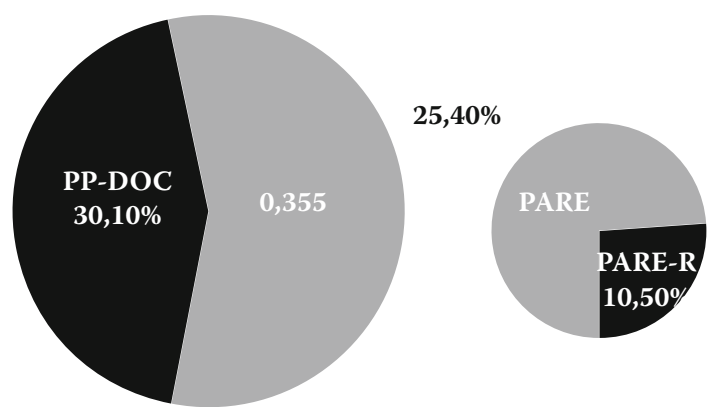

Fuente: elaboración propia

En el código PP-DOC de la docente PAS se logra verificar una participación pedagógica y disciplinar menor en comparación con los docentes CAC y MIY, lo que les permite a los estudiantes participar de manera más activa en las clases de teatro, tanto así que se observa una participación centrada en el estudiante, es decir, más activa e independiente, como menciona Briceño (2013). En los códigos PARE 
y PARE-R evidenciados en el análisis de los estudiantes de la docente PAS se observa que tienen un aprendizaje activo, el cual permite fomentar discusiones en torno al tema, poner en práctica lo aprendido en las clases y ver al alumno como sujeto activo, tal y como señala Briceño (2013).

\section{Relación de frecuencias y códigos de las concepciones y prácticas de los 3 docentes}

En el siguiente gráfico se presentan las relaciones generales de los 3 docentes acerca de la enseñanza y el aprendizaje del teatro que se encontraron, el cual nos muestra, de mayor a menor frecuencia, los principales códigos encontrados.

Figura 7. Relación de la enseñanza y el aprendizaje del teatro en los 3 docentes

\begin{tabular}{|c|c|}
\cline { 2 - 2 } PP-DOC (MIY) & $44,20 \%$ \\
PARE (CAC) & $35 \%$ \\
PP-DOC (MIY) & $30,10 \%$ \\
PARE (PAS) & $25,40 \%$ \\
PARE (MIY) & $21,10 \%$ \\
PP-DOC (CAC) & $21,10 \%$ \\
CET-DIR (CAC) & $14,50 \%$ \\
PARE-R (MIY) & $10,50 \%$ \\
PARE-R (PAS) & $10,10 \%$ \\
\hline
\end{tabular}

Fuente: elaboración propia

Para concluir, el total de códigos y frecuencias relacionados por cada docente es: el de la docente PAS de 172 frecuencias y 10 códigos, del docente CAC de 391 frecuencias y 9 códigos, y del docente MIY de 175 frecuencias y 10 códigos. En estos códigos, organizados de mayor a menor frecuencia, se encuentran el PP-DOC con el 44,20\% con el docente MIY, en comparación con los códigos PARE y PARE-R de los estudiantes, que sumados son el $31.6 \%$, y que indican que posiblemente es un docente que tiene dominio de la materia, como señalan los estudios de Briceño (2013), y en los cuales se cree que es importante esa participación, debido a que el docente orienta una asignatura dirigida a un semestre inicial y es necesario que los estudiantes reciban la información necesaria para su formación.

Enseguida, se observa el código PARE en el docente CAC, con una frecuencia de $35 \%$, en la que puede verse la alta participación de los estudiantes en la clase que, frente a la sumatoria de los códigos PP-DOC y CET-DIR con un $35.6 \%$, evidencia una participación bilateral, como menciona Jaramillo (2013), que crea, de esta manera, un código CET-EB, el cual evidencia que la enseñanza se da de manera equilibrada con los estudiantes, pues ayuda a potencializar de manera sensible, creativa y simbólica al estudiante y al docente. Además, es posible que en esa participación bilateral se encuentre una preocupación centrada en el estudiante.

Posteriormente, se observa el código PP-DOC de la docente PAS con un 30.1\% de frecuencias, en contraste con los códigos PARE y PARE-R de los estudiantes que suman un $35.5 \%$. Se cree que la participación bilateral es un poco más alta, lo cual revela una posible autonomía y apropiación del tema por parte de los estudiantes, seguramente por el conocimiento obtenido en su proceso formativo como estudiantes de sexto semestre y, por lo tanto, todavía se puede observar una participación centrada en el estudiante por parte de la docente, es decir, la participación de los estudiantes es más activa.

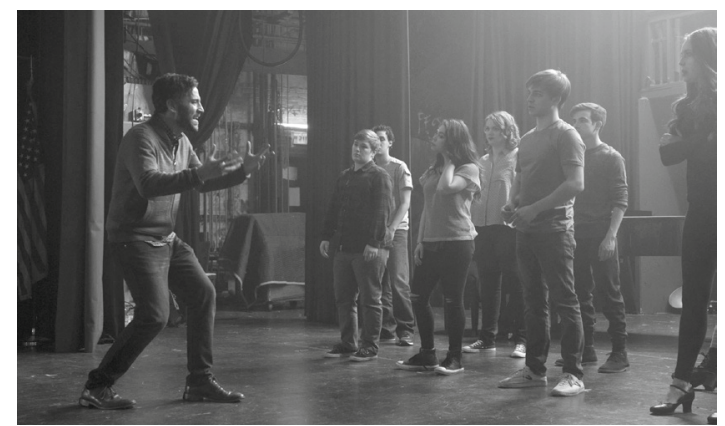




\section{Conclusiones y discusión}

\section{En cuanto a las concepciones}

El teatro se reconoce como una disciplina del arte, que se debe abordar desde el cumplimiento de normas. La enseñanza es, así, un proceso teórico y práctico en el que los acuerdos son importantes y obligan al docente a estar actualizado según sus ideas y preocupaciones específicas frente al teatro.

En cuanto al aprendizaje, se parte de la comprensión del ser humano y de su entorno, los cuales busca encauzar al estudiante para obtener autonomía y apropiación de conceptos teatrales en el ejercicio del mismo, aprovechando el conocimiento de los estudiantes y compañeros de trabajo de manera bilateral.

\section{En cuanto a la práctica}

La docente PAS utiliza didácticas teóricoprácticas que permiten desarrollar habilidades y destrezas en los estudiantes que parten de una particularidad de tipo sensorial propia de sus enseñanzas, diferencia el rol del docente y el de director teatral; finalmente, el rasgo más importante es el dominio de la clase frente a la autonomía de los estudiantes.

El docente CAC tiene la particularidad de enseñar desde la práctica y lo logra desde el rol de director teatral, aunque eso no quiere decir que no tenga clara la diferencia entre ser docente y director. Entre sus principales preocupaciones se encuentran la voz para el teatro y la indagación de textos teatrales para el proceso autónomo del estudiante, por lo que utiliza didácticas propias del teatro para enseñar e invita y motiva a los estudiantes a mejorar sus prácticas educativas desde la práctica y la teoría.

El docente MIY se preocupa mucho por el manejo del cuerpo en escena, sus didácticas provienen de la danza en su gran mayoría, de manera que invita a los estudiantes a trabajar en lo teórico para lograr lo práctico. Del mismo modo, tiene dominio de la materia, lo que permite ver su interés por mejorar su práctica pedagógica; sin embargo, no es claro si el estudiante debe participar más en estas clases y si estos aspectos son necesarios para estas asignaturas, lo que posiblemente derivará en futuras investigaciones sobre estos elementos.

\section{En cuanto a la relación entre las concepciones y la práctica}

En el profesor CAC se encuentra una relación entre lo pedagógico y disciplinar, para quien hay una relación directa entre la dirección teatral como didáctica para la enseñanza del teatro; también se evidencia la preocupación para que sus estudiantes aprendan desde lo teórico-práctico y lo reflexivo, además de estar presto a nuevas propuestas hechas por los estudiantes, lo que permite que se dé un aprendizaje bilateral.

En la profesora PAS se encuentra una relación con el profesor CAC en cuanto a la dirección teatral, ya que se encuentran en procesos de montaje teatral, de ahí que obliguen a los estudiantes a utilizar este recurso como forma de enseñanza. Se resalta su didáctica y particularidad sensorial en el proceso enseñanza-aprendizaje, en el que los acuerdos hacen parte del desarrollo normal de su clase.

En el docente MIY se encuentra que se centra en el estudiante y tiene interés por mejorar sus estrategias pedagógicas desde la empatía con ellos, para brindar herramientas de mejora en su proceso educativo desde el abordaje de textos que enriquecerán la práctica teatral de los participantes.

\section{Conclusiones}

Finalmente, esta investigación ha podido identificar que las concepciones de los tres docentes son bastante diferentes, pues están 
marcadas por sus experiencias de vida. Los tres maestros tienen un dominio claro sobre lo disciplinar y sobre la importancia de lo que significa hacer teatro. La fluidez demostrada en las entrevistas es evidente cuando se indaga por estos aspectos teatrales. Frente a la enseñanza y el aprendizaje, se encuentra que estas concepciones están cargadas de impresiones personales y ubican al estudiante como el centro del proceso de aprendizaje, sin embargo, el uso del lenguaje sobre estas dos últimas variables es coloquial. La reflexión se centra en la importancia de abordar el componente práctico de las clases y no se encuentra un argumento pedagógico avanzado sobre estas temáticas. Hay conocimiento adquirido con las vivencias que han tenido durante su trayectoria como docentes, que son muy pertinentes para lo que realizan, pero valdría la pena, en investigaciones futuras, indagar más en los conocimientos que poseen a fondo sobre estos elementos psicopedagógicos sobre la enseñanza y el aprendizaje, que en esta investigación han quedado expresadas como riquezas propias de la vida de cada maestro; sin embargo, no se alcanza a indagar qué tan cercanas están estas concepciones a conceptos formales que debería manejar un docente que enseñanza en una licenciatura en artes escénicas.

\section{Referencias}

Alfonso, M. (2012). Aportes a la construcción de la identidad profesional del profesor de teatro. Revista Folios, 35, 3-15.

Alfonso, M. (2013). De juegos didácticos en la formación profesoral de teatro: intentos de construcción de un campo profesional. Pensamiento, palabra y obra, 10, 34-55.

Alfonso, M. (2013b). La construcción del saber profesional del profesor de teatro. Estudio sobre la emergencia de praxeologías docentes en la formacion superior (tesis doctoral). Universidad Pedagógica Nacional [UPN], Bogotá.

Alfonso, M. y Rickenmann, R. (2012). La construcción y difusión del docente en las prácticas de formación superior: tensiones y prácticas de referencia de teatro en la saber profesional del artista formación de profesorado. Pensamiento, Palabra y obra, 8, 88-98.

Bajardi, A. y Álvarez Rodríguez, D. (2013). Contribuciones de la educación artística a la construcción de la identidad profesional docente: competencias básicas y comunicativas. Historia y Comunicación Social, 18, 615-626.
Barbosa Sánchez, A. (2016). Educación y arte para la sustentabilidad. Universidad Autónoma del Estado de Morelos, 1-9.

Barco, J. M., Bulla, G. C. y Velásquez, G. A. (2015). Educación de educadores en artes visuales, un lugar de sentido para la educación artística. Pensamieto, Palabra y Obra, $14,114$.

Bertoldi, M., Marano, C. y Rodríguez, M. (2014). La observación como dispositivo en la formación docente de teatro.Una mirada desde dos asignaturas. Trayectoria. Práctica docente en educación artística, 1 , 47-64.

Briceño Martínez, J. (2013). Concepciones y creencias sobre ciencia, aprendizaje y enseñanza de profesores universitarios de ciencias. Revista electrónica de investigación en educación en ciencias, 8(1), 24-41.

Castro, C. (2013). Formación docente en arte. Nuevas configuraciones comunitarias y nuevas demandas a la tarea de enseñar. Revista de educación, 6, 135-148.

Di Bastiano, J. A. y Díaz, C. (2014). El cuerpo docente, lectura y escritura corporal personal. Dramatiza La Plata, 1, 82-85. 
Dimatteo, M. C. (2016). Enfoques de Educación Artística en la formación docente inicial: los contrastes con los diseños curriculares vigentes para la formación de profesores de arte. UNICEN, 9.

Dip, N. y Gómez, M. (2014). Universidad, teatro y mercosur: integrando arte, educación e investigación. Integración y conocimiento, 3(2), 119-128.

Flores Hernández, I. (2013). Personalidad y escuela, cepa de una expresión artística. Calle 14, 8(11), 44-53.

Guercia, C. U. y París, A. C. (2013). Formación de maestros en educación artística y formación artística de maestros. Los patrimonios migratorios en la enseñanza obligatoria. Urpí y Costa, 4, 315.

Jaramillo, E. F. (2013). Estructuras de sentido de la educación artística en la formación de maestros. Revista historia de la educación Colombiana, 301-322.
Kugel, P. (1993). How professors develop as teachers. Studies in Higher Education, 18(3), 315-328.

Martin, J. A. (2016). Theatre Education for Homeschooled Children. Senior Honors Projects, 1-27.

Orce, V. y Mare, M. A. (2016). La formación docente en artes (o el artista que no pudo ser). UNICEN, 10.

Orozco, G. L. (2013). La Práctica docente como núcleo central del desarrollo profesional del profesor universitario. Bogotá D.C: Oficina de publicaciones Universidad de la Salle.

Vieites, M. F. (2014). Educación teatral: nuevos caminos en historia de la educación. Historia de la educación. Revissta interuniversitaria, 33, 77-101.

Vieites, M. F. (2015). De la naturaleza educativa de la educación teatral y de sus rasgos pertinentes. Revista de estudios e investigación en psicología y educación, 4, 1-5. 